\title{
Virtualidad y educación en tiempos de COVID-19. Un estudio empírico en Argentina
}

\section{Virtuality and education in the time of COVID-19. An empirical study in Argentina}

\author{
Cristián David Expósito \\ Consejo Nacional de Investigaciones Científicas y Técnicas-CONICET, Mendoza, Argentina \\ Universidad Nacional de Cuyo, Mendoza, Argentina \\ Roxana Graciela Marsollier (c) \\ Consejo Nacional de Investigaciones Científicas y Técnicas-CONICET, Buenos Aires, Argentina \\ Universidad Nacional de Cuyo, Mendoza, Argentina
}

\begin{abstract}
Resumen
Objetivo: explorar las estrategias, recursos pedagógicos y tecnológicos utilizados por los docentes en el modelo de educación virtual implementado durante el confinamiento preventivo y cierre de establecimientos educativos dispuesto ante la situación de emergencia sanitaria mundial por la COVID-19. Método: se trabajó con un diseño metodológico cuantitativo de tipo descriptivo correlacional. Participaron del estudio 777 personas, en su mayoría docentes, que representan a instituciones de distintos niveles educativos de Mendoza, Argentina. Resultados: el análisis de los datos evidenció las desigualdades existentes en cuanto al uso de tecnologías y recursos pedagógicos digitales. Dichas diferencias se manifiestan en relación con el tipo de gestión, el nivel educativo, la situación socioeconómica de los alumnos, el rendimiento académico y el apoyo por parte de la familia del estudiante. Discusión y Conclusiones: los resultados obtenidos ponen en evidencia las desigualdades socioeducativas de los estudiantes. Es necesario continuar profundizando en la comprensión de los condicionantes y las posibles consecuencias que traerán, para el sistema educativo, estas experiencias pedagógicas en aislamiento social.
\end{abstract}

Palabras clave: COVID-19, pandemia, educación virtual, desigualdad, brecha digital, docentes, necesidades educativas.

\begin{abstract}
Objective: to explore the strategies and the pedagogical and technological resources used by teachers in the virtual education model applied during the preventive quarantine and closure of educational institutions as a result of the global health emergency caused by COVID-19. Method: a quantitative descriptive correlational design was applied. Participants (777 individuals) were mostly teachers representing institutions of different educational levels in Mendoza, Argentina. Results: data analysis showed existing inequality in terms of the use of technology and digital pedagogical resources. Such differences were evidenced in relation to the type of management, students' educational level, socio-economic status, academic performance, as well as their family's support. Discussion and Conclusions: the obtained results show socio-educational inequalities among students. Further understanding the determinants and possible consequences of these pedagogical experiences during quarantine with regard to the educational system is needed.
\end{abstract}

Keywords: COVID-19, pandemic, virtual education, inequality, digital gap, teachers, educational needs. 


\section{Introducción}

La importancia que ha adquirido la educación online en el 2020 a es un hecho sin precedentes que marcará un antes y un después en las prácticas pedagógicas y en los sistemas educativos actuales a nivel global. Asimismo, se ha puesto en evidencia las desigualdades sociales, culturales y económicas de más de 180 países que han sido víctimas de la pandemia por COVID-19 (Bravo-García y Magis-Rodríguez, 2020).

La necesidad y urgencia que trajo consigo la crisis sanitaria actual, hizo que los gobiernos cerraran las puertas de las instituciones educativas como una medida para mitigar los efectos de la pandemia, afectando así al $94 \%$ de los estudiantes a nivel mundial (UNESCO, 2020). Sin embargo, la llama de la educación no puede apagarse y aún en este contexto de extrema emergencia, debe ser garantizada como un derecho humano fundamental (Amuchástegui, et al., 2017).

El reto de los sistemas educativos en los últimos meses ha sido mantener la vitalidad de la educación y promover el desarrollo de aprendizajes significativos. Para ello, ha contado con dos aliados claves: sus docentes y la virtualidad, en términos más precisos, los docentes a través de la virtualidad. Esto ha representado un desafío sin precedentes, ya que la mayoría de los profesores tuvieron que generar sus propios aprendizajes para trabajar en entornos virtuales y, a la vez, fueron los responsables de enseñar a sus estudiantes a manejarse en ese espacio (Bonilla-Guachamín, 2020). Hasta ahora, en términos generales, la educación virtual estaba más bien reservada a experiencias aisladas que aportaban estrategias innovadoras de enseñanza y aprendizaje de manera complementaria a la educación presencial. De hecho, desde hace varias décadas se trabaja en la incorporación de las Tecnologías de la Información y la Comunicación (TIC) como herramientas puestas al servicio de la educación, lo que representa una revisión de los procesos de enseñanza y aprendizaje tradicionales, y un desafío para el rol docente (Almirón y Porro, 2014).

La educación virtual ha sido definida como la educación a distancia a través del ciberespacio, posible mediante la conexión y uso de internet, que no necesita de un tiempo y espacio específicos, que permite establecer un nuevo escenario de comunicación entre docentes y estudiantes (Bonilla, 2016).

Para que una modalidad de educación virtual sea de calidad, debe contemplar ciertos requisitos, tales como: contar con los recursos tecnológicos adecuados y el servicio necesario para acceder al programa educativo; que la estructura y el contenido del curso virtual ofrezcan un valor formativo; que se realicen aprendizajes efectivos y que sea un ambiente satisfactorio tanto para los estudiantes como para los profesores (Marciniak y Gairín-Sallán, 2018). 
Así, la educación de nuestros días se ha valido de entornos virtuales de aprendizaje, puestos a disposición por entidades gubernamentales o plataformas institucionales que casi no funcionaban y de pronto alcanzaron el máximo de su capacidad. Pero, además, otros recursos fueron de gran importancia, principalmente el uso de redes sociales y el fortalecimiento de comunidades de aprendizaje. Con respecto a las redes sociales, siempre hubo cierto reparo en su utilización o potencialidad y una tendencia a acentuar más los obstáculos y riesgos que las ventajas y oportunidades que ofrecían los grupos virtuales, no solo educativos, sino también sociales; sin embargo, con el surgimiento de la pandemia, se han convertido en un recurso altamente valorado. (Robles y Sato, 2020). Con relación a las comunidades de aprendizaje, las mismas pueden darse a nivel institucional con la finalidad de favorecer la capacitación y colaboración entre los docentes (Vaillant, 2017), lo que en estos momentos ha significado que no se sientan solos y estén preparados para enfrentar los desafíos.

Se busca que los entornos virtuales de aprendizaje estén basados en la interpretación y solución de problemas (Gutiérrez-Rodríguez, 2018); que además sean activos y colaborativos (Guitert y Pérez-Mateo, 2013; Anso, et al., 2016; Silva, 2017); que sean afectivos y gamificados (Melo-Solarte y Díaz, 2018), entre otros aspectos. Sin embargo, a raíz de la situación de pandemia, cada docente con sus estudiantes, han generado entornos de aprendizaje de características singulares. Los espacios virtuales, se han ido configurando de distintas formas, mediante los recursos disponibles y las voluntades de toda la comunidad educativa. Cada ambiente formativo se tiñe de valores, de experiencias, de historias de vida de los docentes, que promueven el desarrollo y socialización de las experiencias de sus estudiantes, en vinculación con la familia (Vergel Ortega, et al., 2016).

Cabe destacar que el reto de implementar un sistema educativo a distancia representa diversos desafíos en los distintos niveles, en parte debido a que generalmente la educación virtual ha sido más trabajada en el contexto universitario (Rodríguez-Jiménez, 2009; Silva, 2017; Fernández-Gubieda, 2020). A ello se suman los posgrados y el ámbito de la educación no formal (por ejemplo, a través de capacitaciones o cursos online para docentes, enseñanza de idiomas, cursos de actualización profesional, entre otros). Sin embargo, el resto de los niveles no contaban al momento del cierre de las instituciones por aislamiento social preventivo, con entornos virtuales de aprendizaje que estuviesen operativos y en muchos casos, prácticamente inexistentes. Además, las necesidades educativas son diferentes para cada nivel, la autonomía de los estudiantes varía y, consecuentemente, las estrategias pedagógico-didácticas también son diversas (SánchezMendiola, et al., 2020).

En esta situación marcada por el miedo y la incertidumbre frente a los acontecimientos sanitarios y sus consecuencias sociales, laborales y económicas, la figura del docente ha cumplido un rol fundamental que ha ido mucho más allá de los aspectos pedagógicos. 
Transformó su enseñanza presencial en enseñanza remota desde su hogar y mediatizó materiales didácticos a fin de favorecer el aprendizaje de sus estudiantes (García, 2020). Optar por la virtualidad en educación ofrece un modelo más flexible de enseñanza y aprendizaje, donde las buenas prácticas docentes son las facilitadoras de los procesos educativos mediante el uso de las TIC (Durán-Rodríguez y Estay-Niculcar, 2016).

No obstante, la experiencia actual ha demostrado que el papel docente va más allá de lo pedagógico. Algunos estudios exploratorios anticipan que en este escenario de pandemia el docente no solo debe ser motivador y guía académico, sino también deberá asumir la contención afectiva, ser promotor de resiliencia, asesor emocional y oyente activo; asumiendo, el papel de "guardián de la esperanza de la evolución de la educación" (Villafuerte, Bello, Cevallos y Bermello, 2020, p.149).

Sin embargo, pese al gran esfuerzo colaborativo de toda la comunidad académica en mantener en funcionamiento a la educación, las instituciones no han podido garantizar la igualdad y la justicia social. La situación actual ha puesto en evidencia la desigualdad de oportunidades y condiciones (Tarabini, 2020). En otros términos, la educación virtual ha sido útil para mitigar en parte las consecuencias de la pandemia, ya que al recluir el sistema educativo solo a esta modalidad, y sin una preparación previa a causa de la vertiginosidad con que se dio la crisis, no todos han podido acceder y sacar provecho de ella.

La brecha virtual o digital es una de las problemáticas que más afecta esta situación, por las diferencias en el acceso a los recursos tecnológicos y a la conexión/conectividad a internet, especialmente para los países latinoamericanos (CEPAL, 2020). Para el caso de Mendoza, el porcentaje de alumnos en séptimo grado de nivel primario sin conectividad es del $27,8 \%$ y en quinto año del nivel secundario es de $21 \%$ según los estudios realizados por el Observatorio Argentinos por la Educación (Artopoulos, 2020). A ello se suma la falta de previsión de un sistema de tal envergadura, el cual fue implementado sin la debida planificación, adaptación de las asignaturas y capacitación de docentes y de estudiantes (García-Peñalvo, Abella-García, Corell y Grande, 2020).

Esta situación ha venido a profundizar la desigualdad socioeducativa y la brecha digital, ya que la educación ha quedado supeditada a la virtualidad, donde los recursos tecnológicos y el acceso a internet son imprescindibles (Cabrera, 2020).

En el presente trabajo, se busca identificar las estrategias, recursos pedagógicos y tecnológicos utilizados por los educadores en estos momentos de confinamiento social. Además, indagar sobre las percepciones que tienen los docentes en cuanto al rendimiento académico y apoyo familiar con que cuentan los estudiantes de distintos niveles y modalidades educativas en la provincia de Mendoza, Argentina. 


\section{Método}

\section{Diseño}

Se planteó un diseño metodológico cuantitativo de tipo descriptivo correlacional, donde se analizaron específicamente variables de base e indicadores relacionados al uso de las tecnologías. Además, se indagó sobre los recursos utilizados y las estrategias pedagógicas implementadas por los docentes en la modalidad de educación a distancia impuesta por la pandemia.

\section{Muestra}

La muestra fue establecida de manera intencional o por conveniencia, por lo que es no probabilística. Participaron docentes y directivos de todos los niveles educativos de la Provincia de Mendoza, Argentina, en un total de 777 sujetos. La mayor representatividad está en educación de adultos $(36,16 \%)$, nivel secundario $(31,40 \%)$ y primario $(15,83 \%)$. El $87,91 \%$ corresponde a instituciones de gestión pública. En cuanto a las variables sociodemográficas, predominan las mujeres $(79,51 \%)$, las personas que se encuentran casadas $(51,35 \%)$ y que tienen hijos $(76,19 \%)$. A su vez, la edad promedio de los encuestados es de 43 años.

\section{Instrumentos}

Se elaboró un cuestionario semiestructurado que contempla algunos test, preguntas cerradas y abiertas. Se recolectaron datos sobre variables de base (edad, sexo, estado civil, nivel donde se desempeña, cargo que ocupa, etc.). Por otra parte, se indagó sobre los nuevos escenarios docentes, uso de tecnologías, los recursos y estrategias utilizados en los espacios virtuales.

\section{Procedimiento y análisis de datos}

La aplicación del instrumento fue efectuada mediante Google Formsß. Dicho formulario presenta en primera instancia un acta de consentimiento, donde se expone el objetivo de la investigación y se declara el anonimato y el resguardo del secreto estadístico. La difusión se efectuó mediante correo electrónico y a través de WhatsApp y se solicitó a los participantes que, si estaban de acuerdo con el contenido del cuestionario y evaluaban positivamente el aporte del estudio, reenviaran la encuesta a sus colegas.

El procesamiento de los datos se efectuó mediante el paquete estadístico SPSS ${ }^{\circ}$ 25.0.0. En el tratamiento de los datos cuantitativos, se calcularon las propiedades psicométricas de los cuestionarios a fin de conocer la validez y confiabilidad de los mismos. Posteriormente se trabajó con cálculos estadísticos descriptivos y pruebas paramétricas de varianza (ANOVA), bivariados de asociación $\left(\mathrm{X}^{2}\right)$. 


\section{Resultados y discusión}

\section{Tecnología utilizada}

Teniendo en cuenta la situación de confinamiento domiciliario y la demanda de educación a distancia, se les solicitó a los participantes que indicarán con qué frecuencia utilizaban alguna de las tecnologías propuestas para realizar su tarea pedagógica con sus estudiantes. El instrumento permitió puntuar a cada tecnología mediante una escala Likert de 5 puntos (nunca $=0$ a siempre $=4$ ). Para una mejor interpretación de los datos, se convirtieron los valores a escala 10 obteniendo los siguientes resultados.

Tabla 1.

Tecnología utilizada

\begin{tabular}{|c|c|}
\hline Tecnologías & Puntaje \\
\hline $\begin{array}{l}\text { a) Plataformas virtuales (Moodle } \AA \text {, } \\
\text { Classroom } \AA \text {, etc.) }\end{array}$ & 4,17 \\
\hline $\begin{array}{l}\text { b) Aula virtual Dirección General de } \\
\text { Escuelas }\end{array}$ & 2,66 \\
\hline $\begin{array}{l}\text { C) Videoconferencias }(Z o o m \AA \text {, } \\
\text { MeetHangauts } \AA \text {, etc.) }\end{array}$ & 4,16 \\
\hline d) Grupos WhatsApp $\AA$ & 8,83 \\
\hline e) Foros de debate online & 2,11 \\
\hline 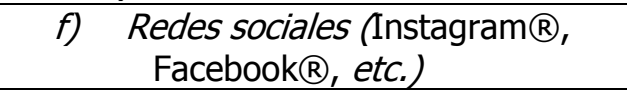 & 3,54 \\
\hline $\begin{array}{l}\text { g) Elaboración de Blog o páginas web } \\
\text { con contenido de estudio }\end{array}$ & 2,24 \\
\hline h) Distribución de material impreso & 2,47 \\
\hline
\end{tabular}

Fuente: elaboración propia, encuesta "Educación en tiempos de Pandemia" 2020.

Como puede observarse en la tabla precedente, la mayor puntuación de frecuencia de uso es para la tecnología WhatsApp con 8,83 pts., despegándose significativamente del resto de las tecnologías. Respecto del material impreso (2,47 pts.), los docentes o algún responsable institucional llevan a las casas de los estudiantes la documentación en formato papel para que éstos lo trabajen. Esta situación es más propia de las zonas rurales donde no hay conectividad o es inaccesible para los alumnos.

Con la intención de reducir variables, se realizó un análisis factorial del instrumento tipo exploratorio con una rotación Varimax. Tanto los resultados de adecuación $(\mathrm{KMO}=0,74)$ y los criterios empíricos obtenidos de la prueba de esfericidad de Bartlett $\left(X^{2}\right.$ Bartlett $=$ $825,934 ; p=0,000)$ son aceptables para realizar el estudio. La matriz de componentes rotados presentó dos factores los cuales quedaron definidos de la siguiente manera: 
- Tecnologías Específicas: refiere al uso de herramientas más adecuadas y complejas, que requieren un conocimiento determinado para su uso (Brasó y Arderiu, 2019). Entre ellas están las plataformas, las videoconferencias, los foros y los blogs o páginas web (4 ítems; $a=0,765$ ).

- Tecnologías Populares: hace referencia al empleo de tecnologías de uso masivo cuyas características son menos específicas y que no demandan ningún tipo de desarrollo para su utilización (Muñíz, 2018). Entre ellas se encuentran el WhatsApp, las redes sociales, el aula virtual de la DGE y los documentos impresos (4 ítems; $a=0,697$ ).

Estos factores fueron relacionados con las variables independientes relevadas por este estudio. En orden a la brevedad solo remitiremos aquellos análisis que presentaron resultados significativos.

La primera de las relaciones relevantes surge con el Tipo de Gestión que es una variable contextual referida a la modalidad de financiamiento de la institución, la cual puede ser privada o estatal. Por ser dicotómica se aplicó una prueba T de Student para muestras independientes obteniendo diferencias significativas en la relación de ambos factores.

Tabla 2.

Tipo de tecnologías en relación con el tipo de gestión institucional

\begin{tabular}{|c|c|c|c|c|c|c|}
\hline & $\begin{array}{l}\text { Tipo de } \\
\text { gestión }\end{array}$ & $\mathbf{N}$ & Media & $\begin{array}{c}\text { Desv. } \\
\text { Desviación }\end{array}$ & $\mathbf{t}$ & $\begin{array}{c}\text { Sig. } \\
\text { (bilateral) }\end{array}$ \\
\hline \multirow[t]{2}{*}{ Específicas } & Estatal & 683 & 2,9502 & 2,27844 & $-7,279$ & 0 \\
\hline & Privada & 94 & 4,7739 & 2,26898 & $-7,302$ & 0 \\
\hline \multirow[t]{2}{*}{ Popular } & Estatal & 683 & 4,4821 & 1,82374 & 4,335 & 0 \\
\hline & Privada & 94 & 3,6104 & 1,85847 & 4,273 & 0 \\
\hline
\end{tabular}

Fuente: elaboración propia, encuesta "Educación en tiempos de Pandemia" 2020.

Este simple análisis paramétrico explica que las escuelas de gestión estatal presentan un mayor manejo de herramientas tecnológicas populares ( $\digamma 4,48)$; sin embargo, las de gestión privada manejan tecnologías más específicas ( $\sigma 4,77)$.

La segunda relación significativa se produce con la variable denominada nivel socioeconómico del estudiante respecto del uso de tecnologías. Debido a que esta variable es categórica se realizó un análisis de varianza unidireccional (ANOVA) para comparar las medias de los factores abordados.

Los resultados obtenidos demuestran la hipótesis de que las tecnologías específicas son utilizadas por los estratos medios, medios altos y altos ( $f=14,845 ; p=0,000$ ); mientras que los estratos inferiores utilizan tecnología denominadas populares ( $f=6,345 ; p=$ $0,000)$. Estos datos se pueden ilustrar de la siguiente manera: 


\section{Figura 1.}

Tipos de tecnologías en relación con el nivel socioeconómico de los estudiantes

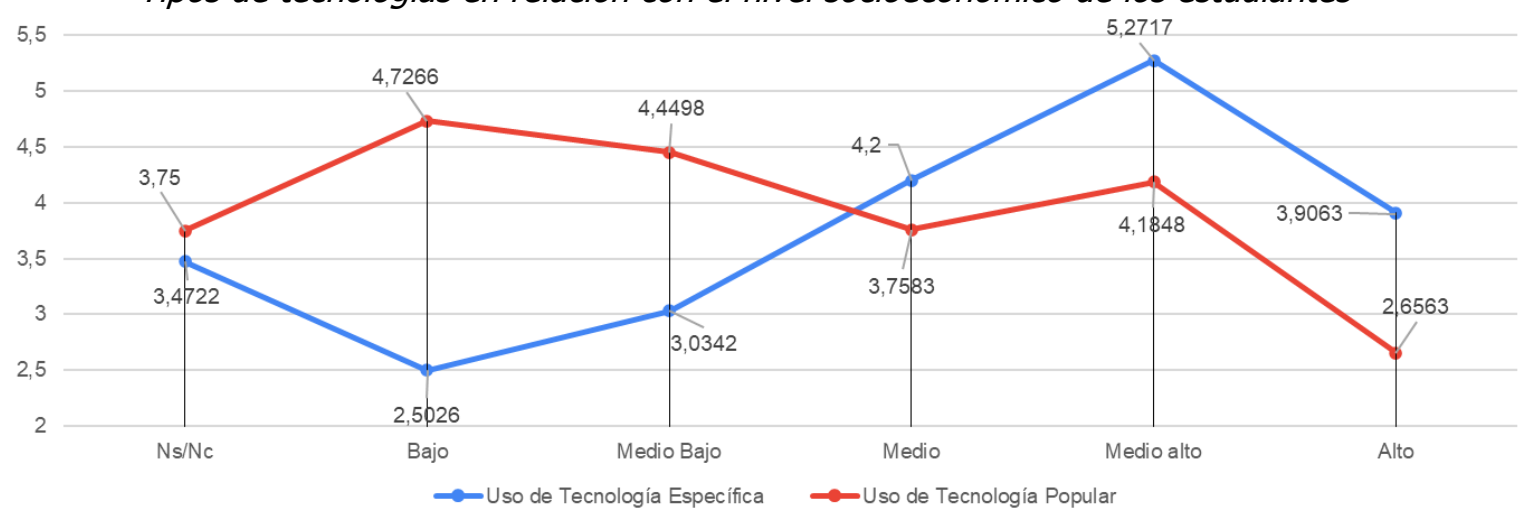

Fuente: elaboración propia, encuesta "Educación en tiempos de Pandemia" 2020.

Claramente se puede observar ( $c f$. Figura 1 ) que los niveles medio bajo y bajo son los que mayor uso hacen de las tecnologías populares y que los niveles medio, medio alto y alto son los que más utilizan tecnologías específicas.

Otra importante relación se produce con la variable denominada nivel educativo que refiere al nivel dentro del sistema educativo donde el docente desarrolla su actividad pedagógica. La prueba resultó significativa para ambos factores; esto sustenta la hipótesis de que las tecnologías utilizadas por los docentes de diferentes niveles no son las mismas. En el caso de las tecnologías específicas los valores presentan un nivel de significación asintótica adecuado $(F=18,574 ; p=0,000)$ al igual que para el factor tecnologías populares $(F=7,245 ; p=0,000)$. Se grafica a continuación estos datos para una mejor interpretación.

Figura 2.

Tipos de tecnologías en relación con el nivel educativo

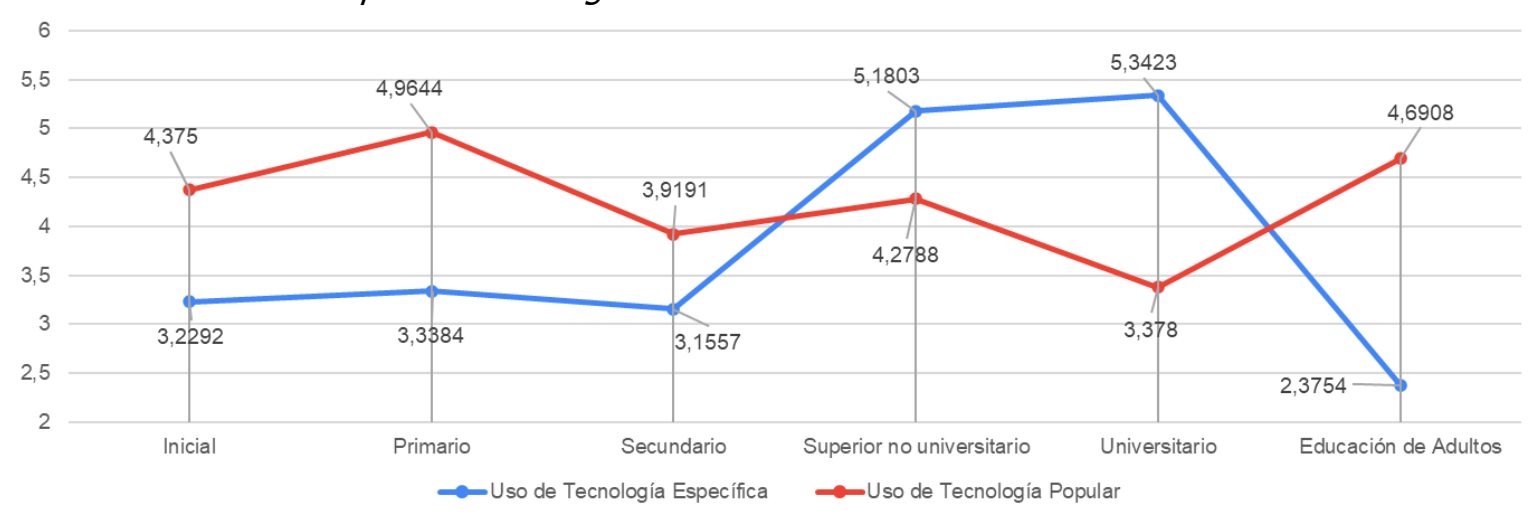

Fuente: elaboración propia, encuesta "Educación en tiempos de Pandemia" 2020. 
Los valores medios de los niveles superiores (Universitario y No Universitario) son los que presentan mayor uso de tecnologías específicas, mientras que los tres niveles obligatorios (Inicial, Primario y Secundario) son los que más uso hacen de tecnologías populares al igual que la Educación de Adultos.

Para un estudio más detallado se realizó un análisis discriminado por nivel y se obtuvieron los siguientes datos:

Tabla 3.

Uso de tecnologías especificas por nivel

\begin{tabular}{ccccccccc}
\hline & $\begin{array}{c}\text { Platafo } \\
\text { rmas }\end{array}$ & $\begin{array}{c}\text { Aula } \\
\text { DGE }\end{array}$ & $\begin{array}{c}\text { Videoconfe } \\
\text {-rencias }\end{array}$ & $\begin{array}{c}\text { Whats } \\
\text { App }\end{array}$ & $\begin{array}{c}\text { Foro } \\
\text { s }\end{array}$ & $\begin{array}{c}\text { Redes } \\
\text { sociale } \\
\text { s }\end{array}$ & $\begin{array}{c}\text { Blog o } \\
\text { web }\end{array}$ & $\begin{array}{c}\text { Material } \\
\text { impreso }\end{array}$ \\
\hline Inicial & 4,375 & 2,5 & 5 & 8,85 & 1,675 & 3,95 & 1,875 & 2,2 \\
\hline Primario & 4,3 & 3,45 & 4,55 & 8,875 & 2,15 & 3,85 & 2,35 & 3,675 \\
\hline Secundario & 4,8 & 2,05 & 3,625 & 8,675 & 2 & 2,975 & 2,175 & 2 \\
\hline Sup. no univ. & 6,55 & 3,5 & 6,2 & 8,225 & 5,05 & 3,55 & 2,925 & 1,825 \\
\hline Universitario & 8,1 & 0,775 & 6,85 & 7,025 & 4 & 4,225 & 2,45 & 1,5 \\
\hline Ed. de Adultos & 2,525 & 3,05 & 3,525 & 9,325 & 1,375 & 3,75 & 2,075 & 2,65 \\
\hline Ed. Especial & 5 & 1,4 & 6,675 & 9,45 & 3,325 & 3,325 & 3,9 & 2,5 \\
\hline
\end{tabular}

Fuente: elaboración propia, encuesta "Educación en tiempos de Pandemia" 2020.

Respecto de las plataformas, los dos niveles de educación superior son los que más uso hacen de ellas. El Aula Virtual de la DGE es utilizada mayormente por el nivel primario y superior no universitario. Las videoconferencias son manejadas principalmente por el nivel de educación especial y los universitarios. Respecto del WhatsApp, son los niveles de adultos y especial los que más destacan. Los foros son trabajados en los dos niveles de educación superior. Las redes sociales captan al nivel universitario e inicial. Los desarrollos de blogs y páginas web son apreciados por el nivel de educación especial y superior no universitario. Por último, el trabajo con material impreso corresponde al nivel primario y educación de adultos. Cabe destacar que ninguna de las tecnologías evaluadas tiene predominancia en la educación secundaria. 
Figura 3.

Uso de tecnologías específicas por nivel
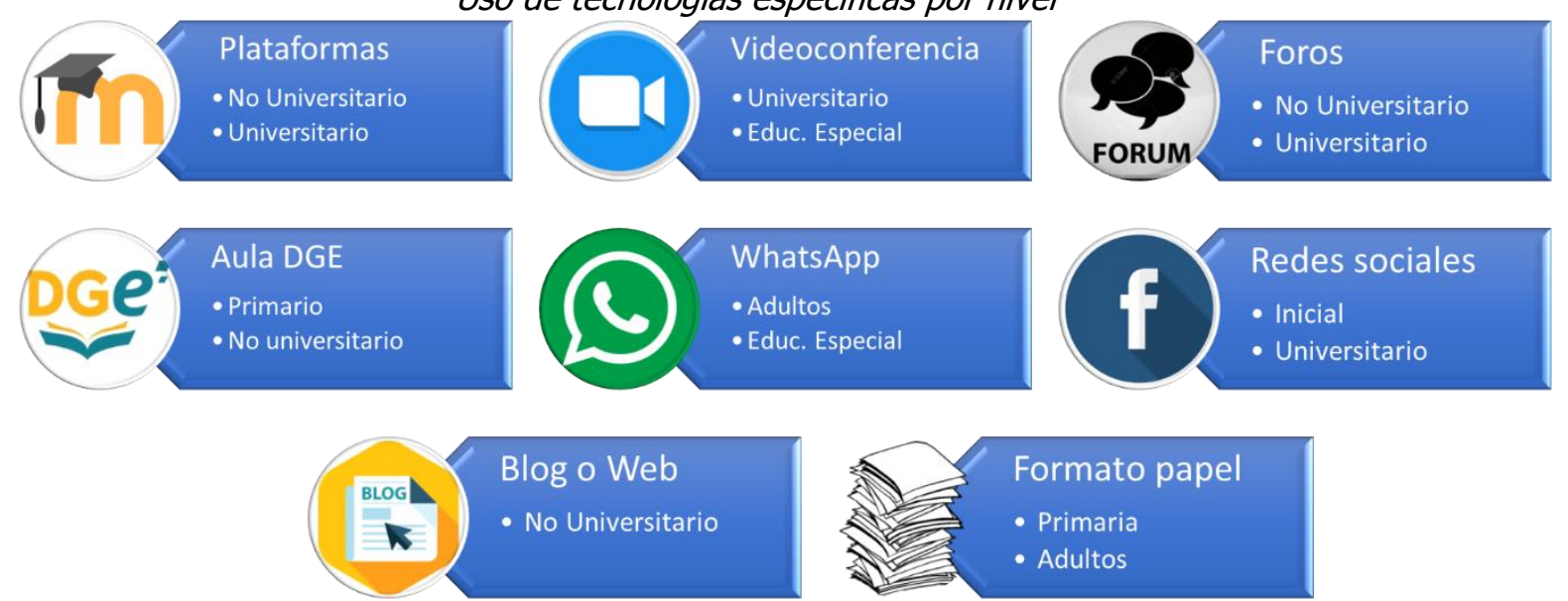

Fuente: elaboración propia, encuesta "Educación en tiempos de Pandemia" 2020.

\section{Recursos pedagógicos digitales}

Siguiendo con el planteo de la labor pedagógica desde casa, se invitó a los participantes a expresar una valoración (categorizada mediante una escala Likert de 5 puntos donde $0=$ no la usé y $4=$ muy positiva) sobre una serie de recursos y estrategias didáctico/pedagógicas utilizadas con sus estudiantes. Para una mejor interpretación también se llevaron los resultados del instrumento a escala 10.

La cantidad de recursos pedagógicos para trabajar de manera remota es muy variada, razón por la cual se han sistematizado en 6 categorías:

1. Clases online: son dictadas en tiempo real por el docente a través de videoconferencias (Guillén y Roig-Vila 2017).

2. Clases grabadas: subidas a $Y o u T u b e \AA$, Vimeo $\mathbb{R}$ o cualquier otra plataforma digital de videos en línea (Horna y Rea, 2017).

3. Digitalización: refiere a aquellos documentos en formato papel que el docente considera imprescindibles para el óptimo desarrollo curricular tales como apuntes de clases o bibliografía seleccionada y los ha llevado a formato digital.

4. Guías de estudio: son herramientas didácticas construidas por el docente en soporte digital transmisibles por mail, WhatsAppR, plataforma o la nube (Liriano, 2016).

5. Evaluación: elaboración de una herramienta o sistema digital que le permita realizar una valoración del rendimiento académico de sus alumnos. 
6. Presentación: diseño de una herramienta didáctica para la facilitación del proceso de aprendizaje de los estudiantes.

Tabla 4.

Lista de recursos pedagógicos digitales

\begin{tabular}{|c|c|}
\hline Tecnologías & Puntaje \\
\hline a) Dictado de clases online & 5,23 \\
\hline b) Grabación digital de clases & 5,61 \\
\hline $\begin{array}{c}\text { c) Digitalización de recursos didácticos } \\
\text { preexistentes }\end{array}$ & 7,40 \\
\hline d) Elaboración de guías de estudio digitales & 7,27 \\
\hline $\begin{array}{c}\text { e) Evaluación a distancia de los contenidos } \\
\text { curriculares }\end{array}$ & 5,92 \\
\hline f) Presentaciones estilo PowerPoint & 5,33 \\
\hline
\end{tabular}

Fuente: elaboración propia, encuesta "Educación en tiempos de Pandemia" 2020.

Las estrategias pedagógicas más destacadas son la digitalización de documentos $(7,4$ pts.) y la elaboración de guías de estudio (7,27 pts.). En último lugar se encuentra el dictado de clases online (5,23 pts.) que se caracteriza por ser uno de los recursos más demandantes de conectividad y ancho de banda.

Este instrumento también fue sometido a un análisis factorial exploratorio con rotación Varimax. El índice de adecuación de la muestra presentó buenos niveles y criterios empíricos viables según la prueba de esfericidad de Bartlett $\left(\mathrm{KMO}=0,773 ; \mathrm{X}^{2}\right.$ Bartlett $=$ $850,428 ; p=0,000)$. La matriz de componentes rotados extrajo dos factores que definimos de la siguiente manera:

- Documentos: este factor reúne aquellos recursos pedagógicos digitales utilizados por el docente para transmitir información a sus alumnos tales como bibliografía, guías de estudio e, inclusive, evaluaciones ( 3 ítems; $a=0,647$ ).

- Clases: se agrupan en este factor los recursos pedagógicos afines con la acción de dictar una clase online ya sea de manera presencial con videoconferencias o diferidas mediante grabaciones. También se incluyen todos aquellos recursos didácticos digitales para la presentación de las clases como un

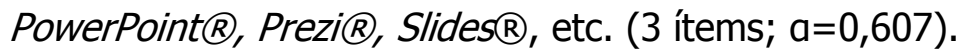

Estos factores fueron sometidos a una serie de análisis paramétricos con las variables contextuales $\mathrm{o}$ independientes relevadas conjuntamente en este estudio. En orden a la brevedad, solo presentaremos las asociaciones significativas.

Se aplicó una prueba $\mathrm{t}$ de Student con la variable referida al tipo de gestión institucional y los resultados obtenidos mostraron una diferencia de medias solo con el 
factor clases:

Tabla 5.

Recursos pedagógicos en relación con el tipo de gestión institucional

\begin{tabular}{rlrrrrr}
\hline \multirow{4}{*}{ Clases } & Tipo de gestión & $N$ & Media & Desv. Desviación & $t$ & Sig. (bilateral) \\
\cline { 2 - 7 } & Estatal & 683 & 5,2806 & 2,66301 & $-3,124$ & 0,002 \\
& Privada & 94 & 6,1968 & 2,68452 & $-3,105$ & 0,002 \\
\hline
\end{tabular}

Fuente: elaboración propia, encuesta "Educación en tiempos de Pandemia" 2020.

Los datos del análisis expresan que existe una diferencia de medias entre ambas gestiones respecto del dictado de clases online. Las escuelas de gestión privada son las que expresan una mejor experiencia con este tipo de herramientas pedagógicas $(\sigma=6,19)$ en comparación con las escuelas de gestión pública ( $\digamma 5,28)$.

Siguiendo con los estudios paramétricos, mediante un análisis de varianza ANOVA se estableció que la variable referida al nivel socioeconómico de los alumnos también presenta relaciones significativas con los factores que refieren al uso de documentos como recurso pedagógico. Para este factor los niveles de varianza son adecuados ( $F=4,816 ; p$ $=0,000)$ al igual que el factor referido al dictado de clases online $(F=3,705 ; p<0,001)$.

Figura 4.

Tipos de recursos pedagógicos en relación con el nivel socioeconómico de los estudiantes

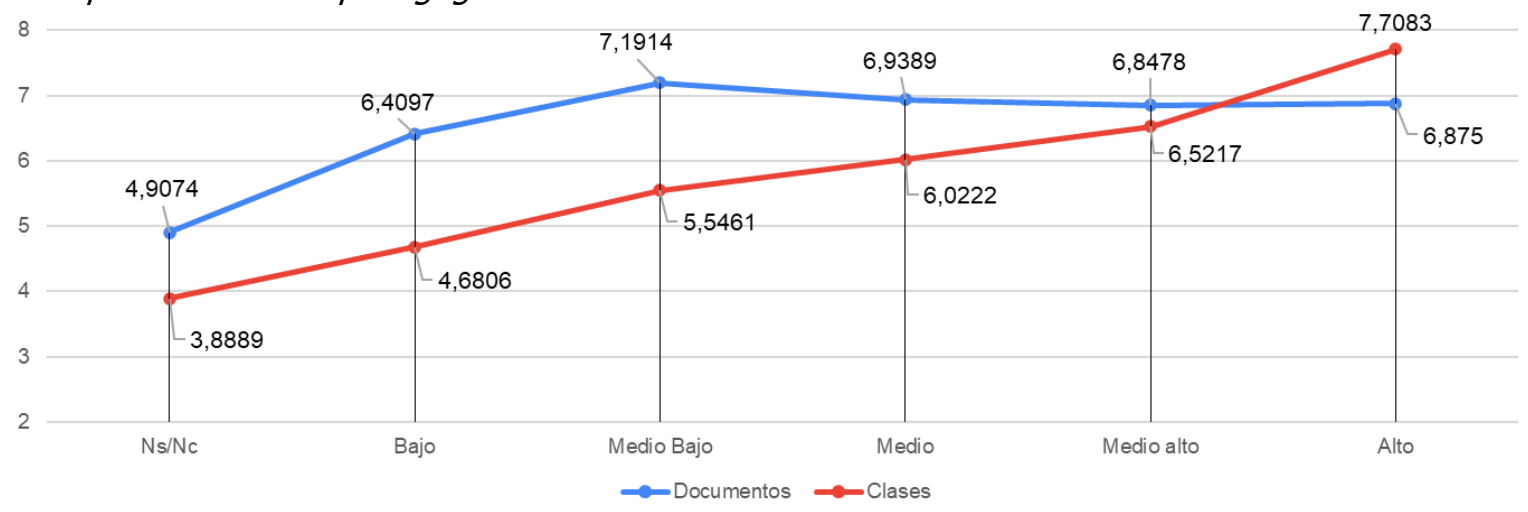

Fuente: elaboración propia, encuesta "Educación en tiempos de Pandemia" 2020.

El análisis indica que se incrementan el uso de recursos referidos al dictado de clases a medida que el estrato socioeconómico de los estudiantes es más elevado; mientras que la variable referida al trabajo pedagógico mediante documentos es mayor en el estrato medio bajo y disminuye paulatinamente a medida que asciende el nivel socioeconómico de los alumnos. Se destaca que, para el nivel bajo, los valores de ambos factores son los más 
pobres de la muestra.

Otra de las variables contextuales que se relaciona con los factores que se analizan, es el nivel educativo donde el docente ejerce su tarea pedagógica. Ambos grupos presentan niveles significativos, en el caso del desarrollo de documentos los valores son $\mathrm{F}=11,816$; $\mathrm{p}=0,000$ y para el dictado de clases online $\mathrm{F}=9,705 ; \mathrm{p}=0,000$.

Figura 5.

Tipos de recursos pedagógicos en relación con el nivel educativo

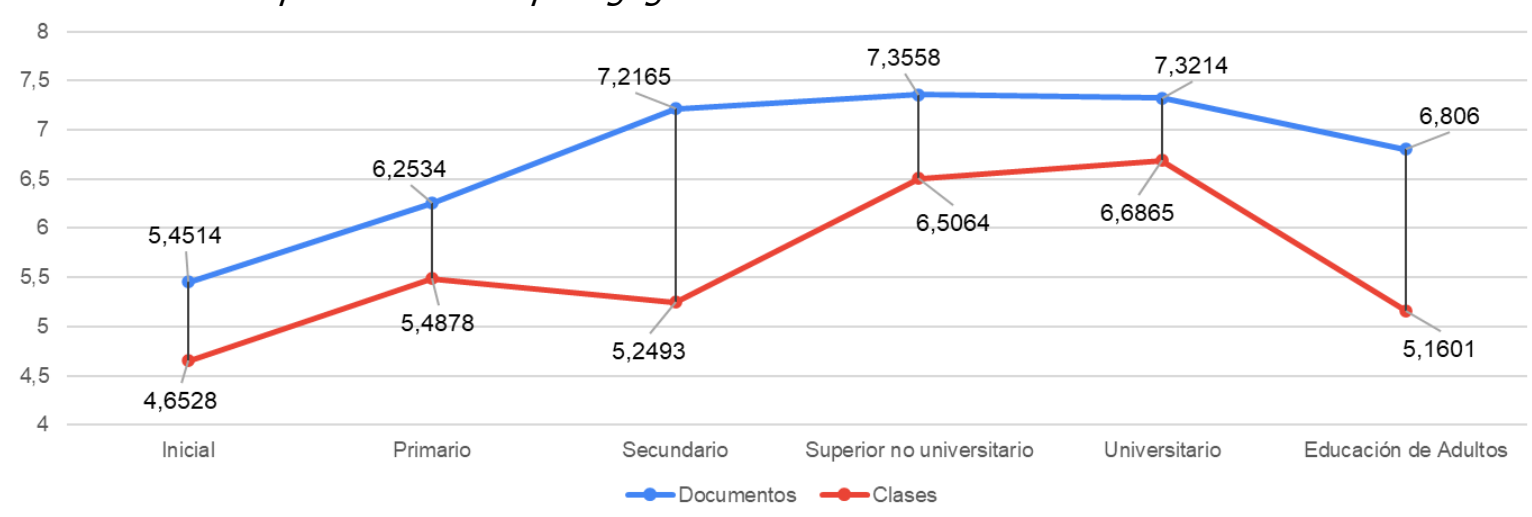

Fuente: elaboración propia, encuesta "Educación en tiempos de Pandemia" 2020.

a medida que el nivel educativo es más elevado, las herramientas pedagógicas utilizada por los docentes son más aprovechadas, presentando el nivel inicial los índices más bajos en ambos recursos. Respecto del nivel secundario y la educación de adultos, se puede observar que existe una disminución en el dictado de clases; por lo tanto, estos niveles reciben mayor flujo de documentos de lectura, estudio y evaluación que de experiencias de interacción personal con el docente (cf. Figura 5). También se destaca que el uso de recursos pedagógicos está focalizado en los documentos de estudio más que en el dictado de clases a nivel general.

Para detallar mejor esta información, se realizó un análisis respecto de las estrategias pedagógicas más utilizadas por el docente dependiendo del nivel educativo en el que ejerce su actividad. 
Tabla 6.

Uso de recursos pedagógicos específicos por nivel

\begin{tabular}{ccccccc}
\hline & $\begin{array}{c}\text { Clases } \\
\text { online }\end{array}$ & $\begin{array}{c}\text { Clases } \\
\text { grabadas }\end{array}$ & $\begin{array}{c}\text { Digitalizació } \\
\mathbf{n}(\text { PDF) }\end{array}$ & $\begin{array}{c}\text { Guías } \\
\text { de } \\
\text { estudio }\end{array}$ & $\begin{array}{c}\text { Evaluació } \\
\mathbf{n}\end{array}$ & $\begin{array}{c}\text { Presentació } \\
\mathbf{n}\end{array}$ \\
\hline Inicial & 4,075 & 5,525 & 6,15 & 5,425 & 4,8 & 4,375 \\
Primario & 4,675 & 6,55 & 7,325 & 6,4 & 5,025 & 5,25 \\
Secundario & 5,325 & 5,1 & 7,65 & 7,725 & 6,3 & 5,325 \\
Sup. no univ. & 6,5 & 6,15 & 7,7 & 8,175 & 6,2 & 6,875 \\
Universitario & 6,6 & 5,95 & 7,625 & 7,55 & 6,775 & 7,5 \\
Ed. de Adultos & 5,075 & 5,525 & 7,2 & 7,275 & 5,95 & 4,875 \\
Ed. Especial & 5,55 & 4,725 & 8,6 & 5,825 & 5 & 5,275 \\
\hline
\end{tabular}

Fuente: elaboración propia, encuesta "Educación en tiempos de Pandemia" 2020.

Las clases online son más utilizadas por los dos niveles de educación superior. Las grabaciones de las clases es una práctica que predomina en docentes del nivel primario y del superior no universitario. La digitalización de documentos también se realiza en la educación superior no universitaria, pero predomina más en educación especial. Con respecto a la elaboración de guías de estudio, es el nivel superior no universitario el que más desarrolla esta actividad seguido por el nivel secundario. La evaluación digital predomina en la formación de grado y en el nivel secundario. Por último, el diseño de presentaciones es una estrategia utilizada mayormente por los dos niveles de educación superior. Cabe mencionar que ninguna de las herramientas evaluadas predomina en la educación de adultos.

Figura 6.

Uso de recursos pedagógicos específicos por nivel

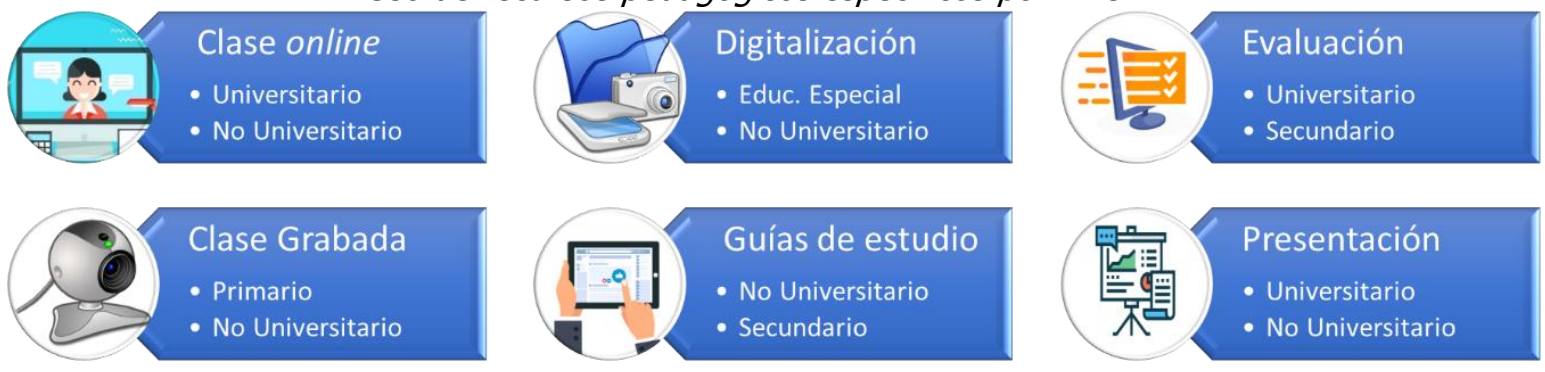

Fuente: elaboración propia, encuesta "Educación en tiempos de Pandemia" 2020.

\section{Rendimiento académico y apoyo familiar}

Se les solicitó también a los participantes que emitieran su opinión respecto del rendimiento académico de sus estudiantes, teniendo en cuenta esta situación atípica para 
la educación. Además, se les invitó a que estimaran el grado de apoyo y contención por parte de los padres o familiares con los que conviven. Los resultados obtenidos son los siguientes:

Tabla 7.

Rendimiento académico de los estudiantes y apoyo familiar

\begin{tabular}{cccc}
\hline & & Porcentaje & Total \\
\hline \multirow{2}{*}{$\begin{array}{c}\text { Rendimiento } \\
\text { Académico }\end{array}$} & Nulo & $0,90 \%$ & \\
& Por debajo de lo normal & $48,91 \%$ & \multirow{2}{*}{$100 \%$} \\
Npormal & $47,36 \%$ & \\
& Por encima de lo normal & $2,83 \%$ & \\
& Sin apoyo & $7,85 \%$ & \\
& Por debajo de lo esperado & $31,40 \%$ & \multirow{2}{*}{$100 \%$} \\
\hline Fuente: elaboración propiar & Normal & $42,73 \%$ & \\
\hline
\end{tabular}

Figura 7.

Rendimiento académico de los estudiantes

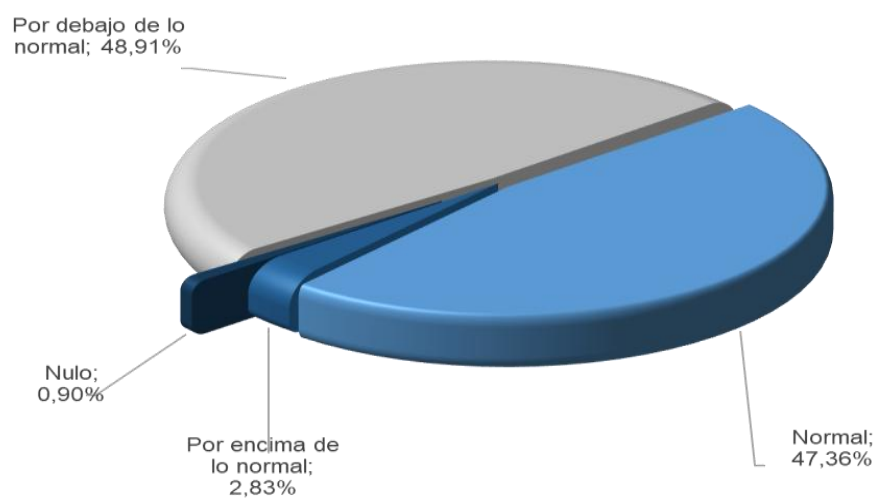

Fuente: elaboración propia, encuesta "Educación en tiempos de Pandemia" 2020.

En términos generales se puede decir que la muestra se dividió en dos mitades, una parte $(48,91 \%)$ sostiene que el rendimiento de sus alumnos está por debajo de lo normal y la otra parte $(47,36 \%)$ plantea que el rendimiento es normal. Los que consideran que el rendimiento se encuentra por encima de lo normal, solo cubren el $2,83 \%$ de la muestra y los que consideran que es nulo apenas llegan al 0,9\%. 
Respecto del nivel de apoyo familiar, el $42,73 \%$ de los docentes estiman que la normalidad se ha mantenido. Casi el tercio $(31,4 \%)$ plantea que en nivel de apoyo es inferior al normal y solo el 7,85\% dice que no existe apoyo familiar. Un $18,02 \%$ de pedagogos resalta que el apoyo superó sus expectativas.

Para un análisis más detallado se relacionaron estas dos variables con el nivel educativo donde el docente lleva a cabo su tarea pedagógica. Los resultados son los siguientes.

Figura 8.

Apoyo familiar de los estudiantes

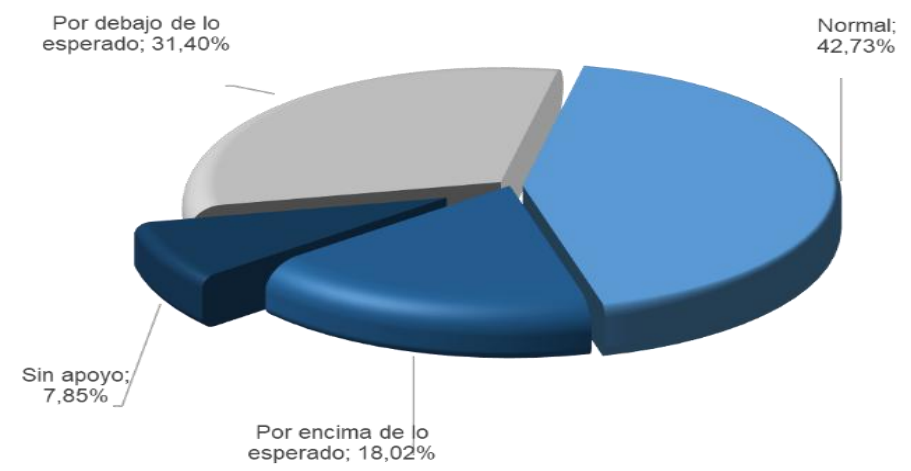

Fuente: elaboración propia, encuesta "Educación en tiempos de Pandemia" 2020

Tabla 8.

Rendimiento académico de los estudiantes y apoyo familiar

\begin{tabular}{ccccccccc}
\hline & & Inicial & Prim. & Sec. & SNU* & Univ. & $\begin{array}{c}\text { Adul } \\
\text { tos }\end{array}$ & Especial \\
\cline { 2 - 9 } & Nulo & $0,0 \%$ & $0,8 \%$ & $1,2 \%$ & $0,0 \%$ & $0,0 \%$ & $1,1 \%$ & $0,0 \%$ \\
Rendimiento & Bajo & $33,3 \%$ & $37,4 \%$ & $59,8 \%$ & $44,2 \%$ & $38,1 \%$ & $48,0 \%$ & $66,7 \%$ \\
Académico & Normal & $66,7 \%$ & $59,3 \%$ & $36,1 \%$ & $51,9 \%$ & $57,1 \%$ & $48,0 \%$ & $33,3 \%$ \\
& Alto & $0,0 \%$ & $2,4 \%$ & $2,9 \%$ & $3,8 \%$ & $4,8 \%$ & $2,8 \%$ & $0,0 \%$ \\
& Sin apoyo & $0,00 \%$ & $3,30 \%$ & $3,70 \%$ & $7,70 \%$ & $\begin{array}{c}16,70 \\
\%\end{array}$ & $\begin{array}{c}13,20 \\
\%\end{array}$ & $0,00 \%$ \\
Apoyo familiar & Bajo & $\begin{array}{c}20,80 \\
\%\end{array}$ & $\begin{array}{c}19,50 \\
\%\end{array}$ & $\begin{array}{c}39,30 \\
\%\end{array}$ & $\begin{array}{c}25,00 \\
\%\end{array}$ & $\begin{array}{c}9,50 \% \\
\%\end{array}$ & $\begin{array}{c}33,80 \\
\%\end{array}$ & $77,80 \%$ \\
& Normal & $\begin{array}{c}37,50 \\
\%\end{array}$ & $\begin{array}{c}48,80 \\
\%\end{array}$ & $\begin{array}{c}38,50 \\
\%\end{array}$ & $\begin{array}{c}53,80 \\
\%\end{array}$ & $\begin{array}{c}64,30 \\
\%\end{array}$ & $\begin{array}{c}40,20 \\
\%\end{array}$ & $0,00 \%$ \\
& Alto & 41,70 & $\begin{array}{c}28,50 \\
\%\end{array}$ & $\begin{array}{c}18,40 \\
\%\end{array}$ & $\begin{array}{c}13,50 \\
\%\end{array}$ & $\begin{array}{c}9,50 \% \\
\%\end{array}$ & $\begin{array}{c}12,80 \\
\%\end{array}$ & $22,20 \%$ \\
\hline
\end{tabular}

*SNU = Superior No Universitario

Fuente: elaboración propia, encuesta "Educación en tiempos de Pandemia" 2020. 
Este análisis pormenorizado permite interpretar que el rendimiento académico del nivel secundario y de educación especial son los que han estado por debajo de las expectativas de los docentes.

Con respecto al apoyo que los estudiantes han recibido en casa, los docentes de nivel inicial y primario plantean que ha sido normal y por encima de lo esperado. Los profesores de nivel secundario, de superior no universitario y de educación de adultos dicen que el apoyo ha sido por debajo de lo normal o normal. Los profesores universitarios sostienen que el acompañamiento es normal y también inexistente. Por último, es necesario resaltar que los docentes de educación especial expresan que este soporte familiar está por debajo de sus expectativas.

Entre los análisis paramétricos realizados destacamos una varianza que presentó niveles significativos con estas dos variables. Los factores referidos a los recursos pedagógicos (documentos y clases) están asociados con las variables de rendimiento académico $(F=10,123 ; p=0,000$ y $F=4,456 ; p=0,004)$ y con el Apoyo familiar $(F=$ 8,605; $p=0,000$ y $F=4,445 ; p=0,004)$.

La hipótesis que argumenta este dato es que, tanto el rendimiento académico como la opinión que tienen los docentes respecto del apoyo familiar va en la misma dirección que el dictado de clases y la elaboración de documentos de estudio y evaluación. Es decir, que tanto el armado de clases como de material de estudio se relaciona con la percepción de respuesta que tienen los docentes sobre el rendimiento académico de sus alumnos y el apoyo de la familia.

\section{Discusiones y Conclusiones}

En este trabajo se indagó sobre las estrategias y recursos, tanto pedagógicos como tecnológicos, utilizados por los docentes de la provincia de Mendoza en el marco del confinamiento preventivo social obligatorio dispuesto por el Poder Ejecutivo de la República Argentina como consecuencia del brote epidémico mundial COVID-19. Los resultados obtenidos expusieron las diferencias sociales y organizacionales existentes respecto a las tecnologías y a los recursos pedagógicos digitales utilizados por los docentes dentro del sistema educativo vigente.

Se puede afirmar, respecto al uso de tecnologías, que la aplicación WhatsApp ha sido la más utilizada por los docentes para comunicarse con sus alumnos y llevar a cabo su tarea pedagógica. Los resultados permitieron identificar dos tipos de tecnologías (Populares y Específicas) y predomina el uso de las tecnologías populares por parte de los alumnos de bajos recursos socioeconómicos, las escuelas de gestión estatal, los niveles de educación obligatoria y en la educación de adultos. Otros autores coinciden en resaltar la importancia del uso de tecnologías populares (en especial WhatsApp) en tanto ha permitido que la 
mayor parte de docentes y estudiantes, especialmente de contextos desfavorecidos, pudieran continuar con el proceso educativo, al menos en un primer momento de la crisis (Kem-Mekah-Kadzue, 2020; Bonilla-Guachamín, 2020). Con referencia a las tecnologías específicas, los resultados indican que prevalece su uso por parte de los estudiantes de nivel socioeconómico medio y alto, en las instituciones de gestión privada y en los niveles superiores (no universitario y de grado) del sistema educativo. Estos datos concuerdan con los aportes de otros estudios sobre los recursos pedagógicos predominantes en el nivel superior, aunque advierten que el uso de estas tecnologías específicas no significa haber superado las desigualdades de acceso a la educación virtual (Alcántara-Santuario, 2020; Mérida Martínez y Acuña-Gamboa, 2020).

Con relación a la implementación de recursos pedagógicos digitales se pudo observar que, al ser tan precipitada esta situación de confinamiento social, los docentes se volcaron a la digitalización de material didáctico preexistente y la elaboración de guías de estudio. A su vez se identificaron dos tipos de recursos (Documentos y Clases) a los que se redujeron los indicadores utilizados. Se pudo constatar que el uso de las clases online como recurso pedagógico está mayormente empleado por los docentes de instituciones de gestión privada, en alumnos de niveles socioeconómicos medios y altos, y en los niveles educativos superiores no universitarios y de grado.

Con referencia al uso de documentación digital como recurso pedagógico se puede mencionar que está más masificado y se utiliza con mayor frecuencia en el nivel secundario y superior (de grado y no universitario). Con relación al aspecto socioeconómico de los estudiantes, el uso de este recurso didáctico es más frecuente en el nivel medio bajo. Señalamos que ambos factores (Documentos y Clases) presentan las puntuaciones inferiores para el sector de bajos recursos. En la misma línea, los aportes de Quiroz-Reyes (2020) sostienen que esta crisis no solo pone en evidencia las desigualdades educativas, sino que también las mismas se han agravado en tanto agregan una nueva desventaja para los estudiantes de los sectores socioeconómicos más desfavorecidos "profundizando la desigualdad en educación al acceder con mayores limitaciones a la educación virtual y en algunos casos, francamente sin acceso al derecho a la educación" (p. 4)

Según expresaron los docentes encuestados, esta situación tan disruptiva para la comunidad educativa influyó negativamente en el rendimiento de los alumnos a lo que se sumaría una disminución del apoyo familiar, en detrimento del rendimiento académico tal como sostienen Muñoz-Moreno y Lluch-Molins (2020). Sin embargo, se ha podido identificar que existe una relación positiva entre el uso de los recursos pedagógico/didácticos digitales por parte del docente, el apoyo familiar del estudiante y su rendimiento académico, que se evidencia en los niveles de educación obligatoria.

En síntesis, los resultados obtenidos confirman las primeras aproximaciones efectuadas en otros contextos, que dan cuenta de que la situación de pandemia puso en evidencia la 
desigualdad de oportunidades educativas entre instituciones públicas y privadas, las diferencias entre quienes tuvieron mejor acceso a los recursos tecnológicos y a internet; las diferencias en el capital cultural de las familias.

\section{Referencias}

Alcántara-Santuario, A. (2020). Educación superior y COVID-19: una perspectiva comparada. En H. Casanova Cardiel (Coord.), Educación y pandemia: una visión académica (pp. 75-82). Ciudad de México: Universidad Nacional Autónoma de México, Instituto de Investigaciones sobre la Universidad y la Educación.

Almirón, M. E., y Porro, S. (2014). Los docentes en la Sociedad de la Información: reconfiguración de roles y nuevas problemáticas. IE Comunicaciones: Revista Iberoamericana de Informática Educativa, (19), 17-31. https://dialnet.unirioja.es/servlet/articulo?codigo $=4794547$

Amuchástegui, G., Valle, M. I. D., y Renna, H. (2017). Reconstruir sin ladrillos: guías de apoyo para el sector educativo en contextos de emergencia. http://repositorio.minedu.gob.pe/handle/MINEDU/5592

Anso, A., Magallan, L., y Vallejos, M. (2016). ¿Actividades grupales en cursos virtuales? Desafíos para el rol docente. En G. Feierherd, (Coordinador). XI Congreso de Tecnología en Educación y Educación en Tecnología (TEyET 2016). Facultad de Informática Ciencias de la Comunicación y Técnicas Especiales, Universidad de Morón.

Argentina.http://sedici.unlp.edu.ar/bitstream/handle/10915/54624/Documento_co mpleto.pdf-PDFA. pdf?sequence=1\&isAllowed =y

Artopoulos, A. (2020) ¿Cuántos estudiantes tienen acceso a Internet en su hogar en Argentina? Observatorio Argentinos por la Educación. https://argentinosporlaeducacion.org/informes

Bonilla, L. A. G. (2016). Deliberación entorno a la Educación Virtual. Interconectando Saberes, (1), 77-89. http://is.uv.mx/index.php/IS/article/view/1112

Bonilla-Guachamín, J. A. (2020). Las dos caras de la educación en el COVID-19. CienciAmérica, 9(2), 89-98. http://dx.doi.org/10.33210/ca.v9i2.294

Brasó, J., y Arderiu, M. (2019). Herramientas tecnológicas para el seguimiento del alumnado en la FP dual. Revista Prácticum, 4(2), 77-94. https://doi.org/10.37042/practicum.2019.4.2.5 
Bravo-García, E., y Magis-Rodríguez, C. (2020). La respuesta mundial a la epidemia del COVID-19: los primeros tres meses. Boletín sobre COVID-19 Salud Pública y Epidemiología, $\quad 1(1), \quad 3-8 . \quad$ http://dsp.facmed.unam.mx/wpcontent/uploads/2013/12/COVID-19-No.1-03-La-respuesta-mundial-a-la-epidemiadel-COVID-19-los-primeros-tres-meses.pdf

Cabrera, L. (2020) Efectos del coronavirus en el sistema de enseñanza: aumenta la desigualdad de oportunidades educativas en España. Revista de Sociología de la Educación-RASE, 13(2), 114-139. https://doi.org/10.7203/RASE.13.2.17125

CEPAL. (2020). Las oportunidades de la digitalización en América Latina frente al Covid19. https://repositorio.cepal.org/handle/11362/45360

Durán-Rodríguez, R., y Estay-Niculcar, C. (2016). Formación en buenas prácticas docentes para la educación virtual. RIED. Revista Iberoamericana de Educación a Distancia, 19(1), 209-232. https://doi.org/10.5944/ried.19.1

Fernández-Gubieda, S. (2020). Docencia Rubic: aprendizajes de la enseñanza universitaria en tiempos de la Covid-19. https://www.unav.edu/documents/24640148/26406619/RubicSueltas.pdf

García, M. D. (2020). La docencia desde el hogar. Una alternativa necesaria en tiempos del COVID 19. Polo del Conocimiento: Revista científico-Académica Multidisciplinaria, 5(4), 304-324. https://doi.org/10.23857/pc.v5i4.1386

García-Peñalvo, F. J., Abella-García, V., Corell, A., y Grande, M. (2020). La evaluación online en la educación superior en tiempos de la COVID-19. Education in the Knowledge Society (EKS),(21), 1-26. http://dx.doi.org/10.14201/eks.23086

Guillén, A. y Roig-Vila, R. (2017). Escenarios online para el aprendizaje de un instrumento musical: sinopsis de algunas investigaciones. Notandum, 20(44-45), 43-52. http://dx.doi.org/10.4025/notandum.44.5

Guitert, M., y Pérez-Mateo, M. (2013). La colaboración en la red: hacia una definición de aprendizaje colaborativo en entornos virtuales. Teoría de la Educación. Educación y Cultura en la Sociedad de la Información, 14(1), 10-31. http://hdl.handle.net/10366/121846

Gutiérrez-Rodríguez, C. A. (2018). Fortalecimiento de las competencias de interpretación y solución de problemas mediante un entorno virtual de aprendizaje. Revista de Investigación, Desarrollo e Innovación, 8(2), 279-293. 
http://132.248.161.133:8080/jspui/handle/123456789/4249

Horna, K., y Rea, W. (2017). Uso de la Comunicación Audiovisual en las Clases de los Estudiantes del 5to Grado de la IE 20318 José Antonio Macnamara, Huacho 2016 [Tesis de grado, Universidad Nacional José Faustino Sánchez Carrión, Perú].http://repositorio.unjfsc.edu.pe/handle/UNJFSC/469

Kem-Mekah-Kadzue, O. (2020). Enseñanza en línea durante la crisis del Covid-19 en la educación universitaria camerunesa: logros y desafíos. Ehquidad. International Welfare Policies and Social Work Journal,(14), 57-74. http://dx.doi.org/10.15257/issn.2386-4915

Liriano, R. (2016). El incremento de las competencias tecnológicas en los estudiantes que ingresan a la Educación a Distancia. INTEC Sobre el papel de los estudiantes en los Estudios Generales, (23), 307-326. http://rai.uapa.edu.do:8080/xmlui/bitstream/handle/123456789/411/Trabajo\%20en $\% 20$ memoria\%20de\%20evento\%202016. pdf? sequence $=1$ \&isAllowed $=$ y\#page $=314$

Marciniak, R., y Gairín-Sallán, J. (2018). Dimensiones de evaluación de calidad de educación virtual: revisión de modelos referentes. RIED. Revista Iberoamericana de Educación a Distancia, 21(1), 217-238. https://doi.org/10.5944/ried.21.1.16182

Melo-Solarte, D. S., y Díaz, P. A. (2018). El aprendizaje afectivo y la gamificación en escenarios de educación virtual. Información tecnológica, 29(3), 237-248. http://dx.doi.org/10.4067/S0718-07642018000300237

Mérida-Martínez, Y., y Acuña-Gamboa, L. A. (2020). Covid-19, Pobreza y Educación en Chiapas: Análisis a los Programas Educativos Emergentes. Revista Internacional de Educación para la Justicia Social, https://revistas.uam.es/riejs/article/view/12435

Muñíz, R. (2018). Las Tecnologías Apropiadas ¿Un Cambio de Paradigma o una Utopía? Tekhné, 21(1), 87.http://revistasenlinea.saber.ucab.edu.ve/temas/index.php/tekhne/article/view/3 547

Muñoz-Moreno, J. L., y Lluch-Molins, L. (2020). Educación y Covid-19: Colaboración de las Familias y Tareas Escolares. Revista Internacional de Educación para la Justicia Social, 9(3), 1-17. https://revistas.uam.es/riejs/article/view/12182

Quiroz-Reyes, C. (2020). Pandemia Covid-19 e Inequidad Territorial: El Agravamiento de 
las Desigualdades Educativas en Chile. Revista Internacional de Educación para la Justicia Social, 9(3),1-6. https://revistas.uam.es/riejs/article/view/12143

Robles, C., y Sato, A. (2020). Grupalidades virtuales. El impacto de la pandemia en los procesos grupales. En La intervención en lo social en tiempos de pandemia. https://www.margen.org/pandemia/textos/robles.pdf

Rodríguez-Jiménez, J. M. (2009). Patrones pedagógicos en educación virtual. Revista de Educación a Distancia (RED). Monográfico X (2009), 1-16. https://revistas.um.es/red/article/view/89321

Sánchez-Mendiola, M., Martínez-Hernández, A. M., Torres-Carrasco, R., Agüero-Servín, M., Hernández-Romo, A., Benavides-Lara, M., Jaimes-Vergara, C. y Redón-Cazales, V. (2020) Retos educativos durante la pandemia de COVID-19: una encuesta a profesores de la UNAM. Revista Digital Universitaria, Aheadofprint, (2020), 1-23. https://www.revista.unam.mx/wp-content/uploads/AOP.pdf

Silva, J. (2017). Un modelo pedagógico virtual centrado en las E-actividades. Revista de $\begin{array}{llll}\text { Educación } & a & \text { Distancia, } & 17(53),\end{array}$ https://revistas.um.es/red/article/view/290021

Tarabini, A. (2020) ¿Para qué sirve la escuela? Reflexiones sociológicas en tiempos de pandemia global. Revista de Sociología de la Educación-RASE, 13(2), 145-155. https://doi.org/10.7203/RASE.13.2.17135

UNESCO (06 de agosto de 2020). El Secretario General de las Naciones Unidas advierte de que se avecina una catástrofe en la educación y cita la previsión de la UNESCO de que 24 millones de alumnos podrían abandonar los estudios. https://es.unesco.org/news/secretario-general-naciones-unidas-advierte-que-seavecina-catastrofe-educacion-y-cita

Vaillant, D. (2017). Directivos y comunidades de aprendizaje docente: un campo en construcción. En Weinsteins y Muñoz (Eds.), Mejoramiento y Liderazgo en la escuela (pp. 263-291). Once miradas. http://dx.doi.org/10.14244/198271993073

Vergel-Ortega, M., Rincón-Leal, O. L., y Cardoza-Herrera, C. A. (2016). Comunidades de aprendizaje y prácticas pedagógicas. Boletín Redipe, 5(9), 137-145. https://dialnet.unirioja.es/servlet/articulo?codigo $=6064925$

Villafuerte, J., Bello, J., Cevallos, Y., y Bermello, J. (2020). Rol de los docentes ante la crisis del COVID-19, una mirada desde el enfoque humano. REFCalE: Revista Electrónica Formación y Calidad Educativa, 8(1), 134-150. http://www.refcale.uleam.edu.ec/index.php/refcale/article/view/3214 